\title{
Postpartum Hysterectomy a Case Report of a Saving Life Surgery
}

\author{
Alfredo de Almeida Cunha, Cláudio de Oliveira Soeiro, Sérgio Araujo Martins Teixeira, \\ Emílio de Assis
}

Hospital Estadual da Mãe, Hospital e Maternidade Therezinha de Jesus, Rocha Sobrinho, Mesquita, RJ, Brasil

Email address:

alfredocunha.iep@hmtjrj.org.br (A. de A. Cunha)

\section{To cite this article:}

Alfredo de Almeida Cunha, Cláudio de Oliveira Soeiro, Sérgio Araujo Martins Teixeira, Emílio de Assis. Postpartum Hysterectomy a Case Report of a Saving Life Surgery. Journal of Gynecology and Obstetrics. Vol. 4, No. 6, 2016, pp. 57-61. doi: 10.11648/j.jgo.20160406.15

Received: July 3, 2016; Accepted: December 5, 2016; Published: January 7, 2017

\begin{abstract}
Postpartum hemorrhage ( $\mathrm{PPH})$ is the most cause of maternal mortality all over the world important. Its prevalence is about 4 cases in 10,000 deliveries. However, the risk of maternal death is nearly $16 \%$ of all maternal deaths in developing countries, especially in Africa. It depends on the resources to stop bleeding, medical and surgical. The first ones are uterotonics medication (prostaglandin, methylergonovine and oxytocin), and the latest professional apt to perform surgical procedures, such as B-Lynch surgery and the peripartum hysterectomy. A woman, 20 years old, GI, P0, A0, with a single pregnancy, 42 weeks of gestational age and 5 prenatal bookings was attended at the emergency on April $12^{\text {th }} 2015$. The next day the patient delivered a male baby. The uterus was soft and at the examination she expelled a great amount of clots. She was prescribed Ringer lactate $500 \mathrm{ml}+$ oxytocin $15 \mathrm{IU}+$ methylergonovine $0.2 \mathrm{mg} \mathrm{IM}+$ misoprostol $800 \mu \mathrm{g}$ per rectum. She continued bleeding and a hysterectomy was performed. The patient was discharge in good condition. The pathology revealed myometrium permeated by trophoblasts (placenta accreta).
\end{abstract}

Keywords: Hysterectomy, Postpartum Hemorrhage, Surgery, Oxytocin, Misoprostol

\section{Introduction}

Postpartum hemorrhage (PPH) is an important cause of maternal mortality. Carolli et al (2008) conducted a systematic review of the prevalence of PPH. After establishing PPH ( $>$ or $=500 \mathrm{~mL}$ blood loss) and severe PPH (SSPH) (>or=1000 mL blood loss) as main outcomes the prevalence of $\mathrm{PPH}$ and SPPH is approximately $6 \%$ and $1.86 \%$ of all deliveries [1]. Maternal mortality due to postpartum hemorrhage (PPH) continues to be one of the most important causes of maternal death worldwide. PPH is a significantly underestimated obstetric problem, primarily because a lack of definition and diagnosis [2, 3].

To quantify the contribution of placenta accreta to the rate of postpartum hemorrhage and severe postpartum hemorrhage, Mehrabadi et al (2015) reviewed all hospital deliveries in Canada (excluding Quebec) for the years 2009 and $2010(\mathrm{~N}=570,637)$ included in a retrospective cohort study using data from the Canadian Institute for Health Information. Placenta accreta included placental adhesion to the uterine wall, musculature, and surrounding organs (accreta, increta, or percreta). Severe postpartum hemorrhage included postpartum hemorrhage with blood transfusion, hysterectomy, or other procedures to control bleeding (including uterine suturing and ligation or embolization of pelvic arteries). Rates, rate ratios, population-attributable fractions (i.e., incidence of postpartum hemorrhage attributable to placenta accreta), and 95\% confidence intervals (CIs) were estimated. Logistic regression was used to quantify associations between placenta accreta and risk factors. The incidence of placenta accreta was 14.4 (95\% CI 13.4-15.4) per 10,000 deliveries (819 cases among 570,637 deliveries), whereas the incidence of placenta accreta with postpartum hemorrhage was 7.2 (95\% CI 6.5-8.0) per 10,000 deliveries. Postpartum hemorrhage among women with placenta accreta was predominantly third-stage hemorrhage (41\% of all cases). Although placenta accreta was strongly associated with postpartum hemorrhage (rate ratio $8.3,95 \%$ CI 7.7-8.9), its low frequency resulted in a small populationattributable fraction $(1.0 \%, 95 \%$ CI $0.93-1.16)$. However, the strong association between placenta accreta and postpartum 
hemorrhage with hysterectomy (rate ratio 286, 95\% CI 226361 ) resulted in a population-attributable fraction of $29.0 \%$ (95\% CI 24.3-34.3). They conclude that placenta accreta is too infrequent to account for the recent temporal increase in postpartum hemorrhage but contributes substantially to the proportion of postpartum hemorrhage with hysterectomy [4].

\section{Case Report}

A woman, 20 years old, GI, P0, A0, with a single pregnancy, 42 weeks of gestational age and 5 prenatal bookings was attended at the emergency on April 12 2015 . The clinical examination revealed $\mathrm{BP}=140 \times 90 \mathrm{mmHg}$. The next day the patient delivered a male baby. After delivery the uterus was soft and at the examination she expelled a great amount of clots. She was prescribed Ringer lactate $500 \mathrm{ml}+$ oxytocin $15 \mathrm{IU}+$ methylergonovine $0.2 \mathrm{mg} \mathrm{IM} .+$ misoprostol $800 \mu \mathrm{g}$ per rectum. As was still bleeding, a hysterectomy was performed. The patient was discharge in good condition on April 17th 2015.

The pathology showed myometrium permeated by trophoblasts (H. E. 40X) (figure 1-2), placenta accreta.

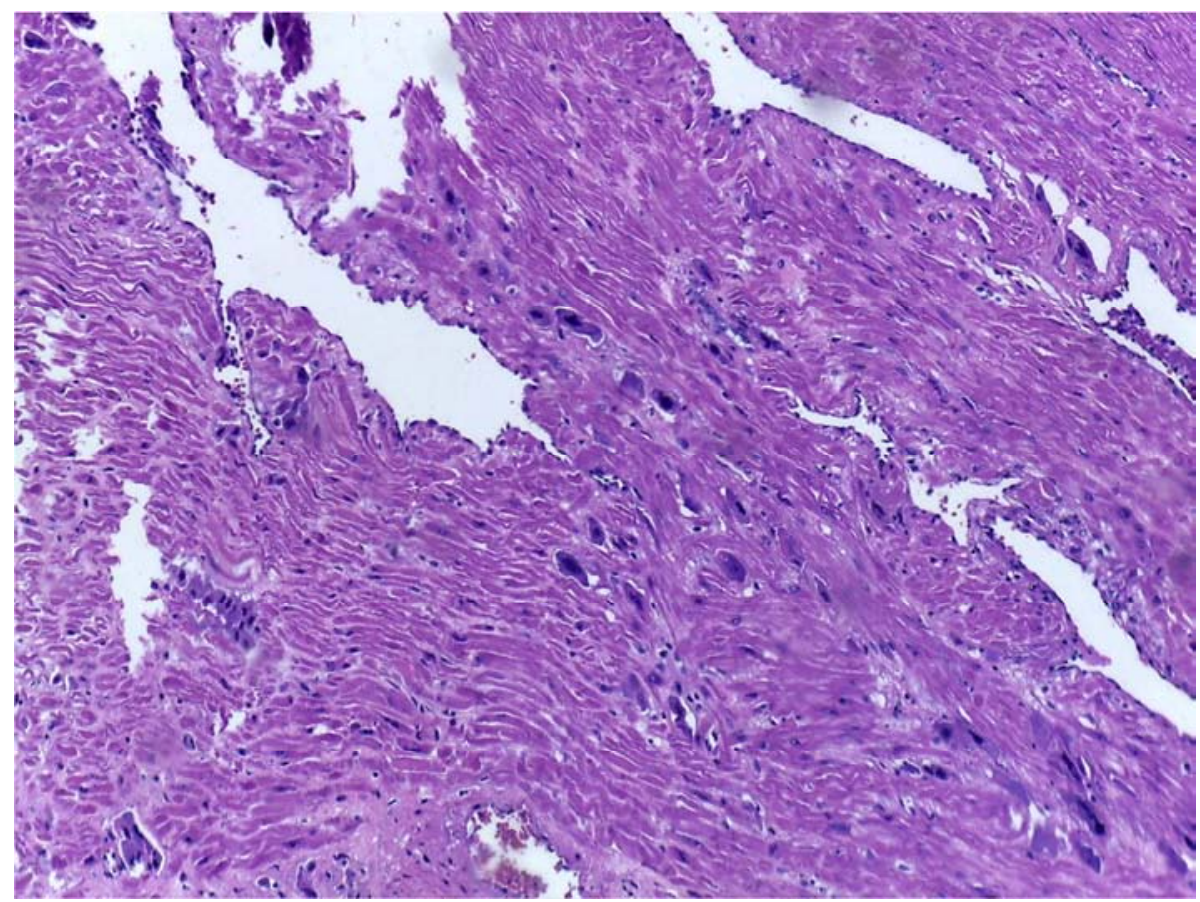

Figure 1. Trophoblast permeating the endometrium.

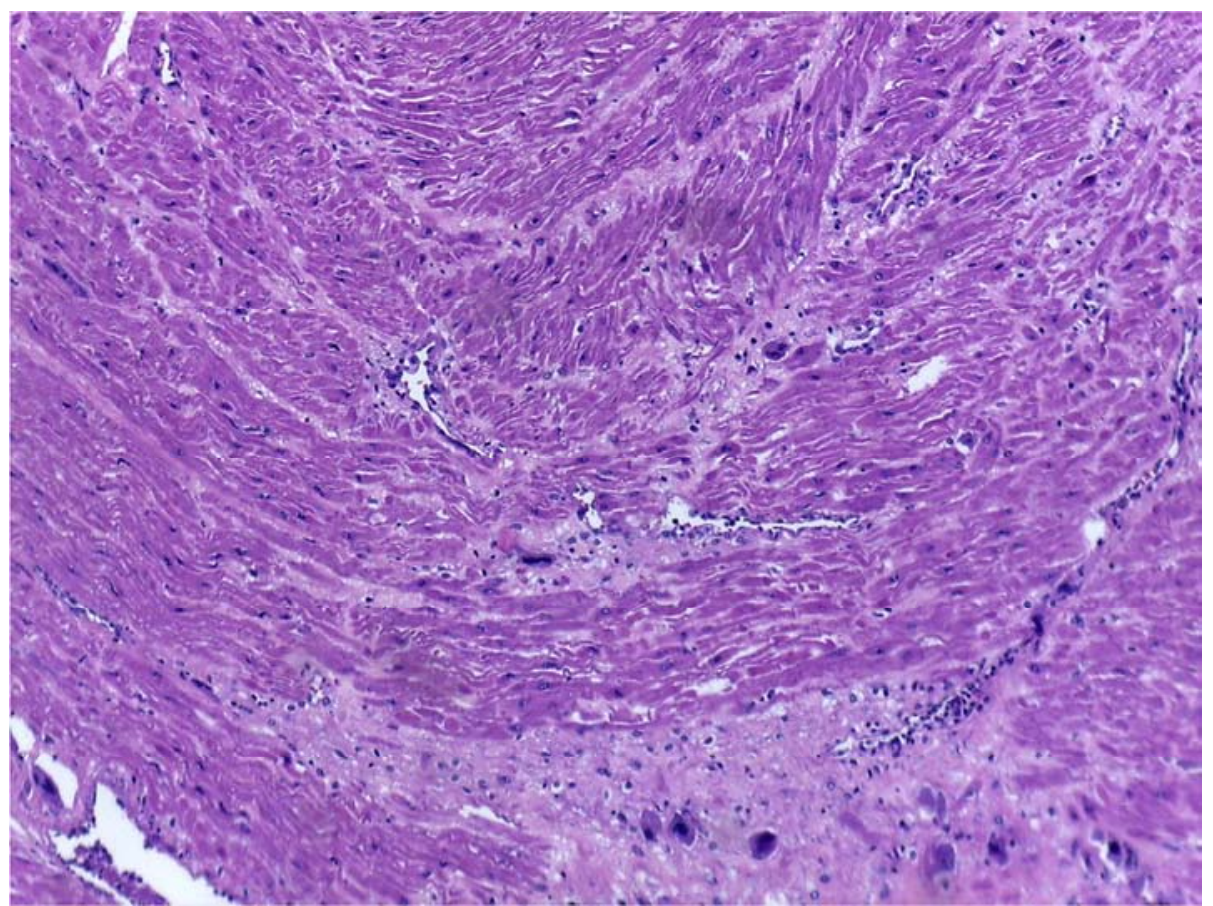

Figure 2. Trophoblast permeating the endometrium. 


\section{Discussion}

Postpartum hemorrhage (PPH) remains one of the leading causes of maternal morbidity and mortality worldwide, although the lack of a precise definition precludes accurate data of the absolute prevalence of PPH. To clarify this question an international expert panel in obstetrics, gynecology, hematology, transfusion, and anesthesiology undertook a comprehensive review of the literature. At a meeting in November 2011, the panel agreed on a definition of severe PPH that would identify those women who were at a high risk of adverse clinical outcomes. The panel agreed on the following definition for severe persistent (ongoing) PPH: "Active bleeding $>1000 \mathrm{~mL}$ within the 24 hours following birth that continues despite the use of initial measures including first-line uterotonics agents and uterine massage." A treatment algorithm for severe persistent PPH was subsequently developed. Initial evaluations include measurement of blood loss and clinical assessments of PPH severity. Coagulation screens should be performed as soon as persistent (ongoing) PPH is diagnosed, to guide subsequent therapy. If initial measures fail to stop bleeding and uterine atony persists, second- and third-line (if required) interventions should be instated. These include mechanical or surgical maneuvers, i.e., intrauterine balloon tamponade or hemostatic brace sutures with hysterectomy as the final surgical option for uncontrollable PPH. Pharmacologic options include hemostatic agents (tranexamic acid), with timely transfusion of blood and plasma products playing an important role in persistent and severe PPH. CONCLUSION: Early, aggressive, and coordinated intervention by health care professionals is critical in minimizing blood loss to ensure optimal clinical outcomes in management of women with severe, persistent PPH [5].

In our study we followed this protocol, except that we jumped from second line to the fourth line, hysterectomy, with the intention of saving the mother's life.

Mehrabadi et al studied all hospital deliveries in Canada $(\mathrm{N}=570,637)$. The incidence of placenta accreta was 14.4 per 10,000 deliveries (819 cases among 570,637 deliveries), whereas the incidence of placenta accreta with postpartum hemorrhage was 7.2 per 10,000 deliveries. Although placenta accreta was strongly associated with postpartum hemorrhage (rate ratio 8.3), its low frequency resulted in a small population-attributable fraction (1.0\%, 95\% CI $0.93-1.16)$. However, the strong association between placenta accreta and postpartum hemorrhage with hysterectomy (rate ratio 286) resulted in a population-attributable fraction of $29.0 \%$ [4].

Diagnosis of abnormalities of placentation other than placenta previa remains a challenge. An improvement might be the implementation of standardized operating procedures for retained placenta which could contribute to a reduction of maternal morbidity [6].

Placenta accreta is characterized by excessive penetration of the villi into the myometrium, which obstructs its correct separation during stage III of labor. That in turn leads to a potentially life-threatening maternal hemorrhage. Until recently this pathology has been a rare occurrence but currently it is fifty times more prevalent. Placenta accreta is associated with high morbidity and the risk of maternal death, even despite advances in ultrasound diagnosis, wellestablished surgical treatment, and multi-disciplinary medical care. A dramatic rise in the rates of Cesarean section and intrauterine surgical procedure is considered to be the main factor responsible for the growing incidence of placenta accreta. It is especially frequent in women after a Cesarean section and with placenta previa covering the lower segment. A Cesarean section, combined with hysterectomy and application of various techniques to limit massive bleeding, is usually performed between 34-36 weeks of pregnancy before the onset of labor [7]

Takebayashi et al reported a case whose pathological examination resembles our case. It showed that implantation site intermediate trophoblasts (ISITs) formed unusually large clumps in the decidua, diagnosed as exaggerated placental site (EPS). EPS is thought to be a condition consisting of an excessive number of ISITs. ISITs are differentiated from a trophoblast lineage in the process of placenta formation. ISITs anchor the placenta to the maternal tissue and are considered to maintain pregnancy, but the postpartum role of these cells remains unclear. Excessive infiltration of ISITs, namely EPS, may cause postpartum atonic uterus. The present case and other reported cases indicate that EPS causes mass formation in the uterus, continuous uterine bleeding, and massive hemorrhage, resulting in hysterectomy [8].

Luo et al give another explanation for our case that is residuals of placenta and fetal membranes (one in 6,688 deliveries in 2015 in our institution). They studied the cause and preventative measures of late postpartum hemorrhage. The conclusion was that placental and fetal membrane residuals are the major cause of late postpartum hemorrhage [9].

The risk for hysterectomy in our institution in 2015 was 4 cases in 6,688 deliveries. Fitzpatrick et al made a research to study the relationship of abnormal placentation and cesarean hysterectomy risk factors. They found that $33.3 \%$ had a cesarean hysterectomy and $41.7 \%$ had post-partum hemorrhage. Also 51.7\% received blood transfusion. The probability of cesarean hysterectomy in patients with abnormal placentation was significantly increased in patients with previous cesarean sections. By decreasing the rate of cesarean section we can decrease the incidence of cesarean hysterectomy and its comorbidities [10].

The present case was managed with a resumed protocol: uterine massage and manual removal of the clots as the first line, oxytocin, methylergonovine and misoprostol as the second line, jumping to the fourth line, hysterectomy, as a heroic measure to save the mother's life. Post-cesarean hysterectomy is the most extensive procedure used during the postpartum stage. This is an indicator of quality substantially 
associated with extreme obstetric morbidity [11]. Butwick et al (2015), even considering that most cases of postpartum hemorrhage respond to first-line therapy with uterine massage and oxytocin administration, second-line uterotonics including methylergonovine and carboprost are integral for the management of refractory uterine atony. Despite their ubiquitous use, it is uncertain whether the risk of hemorrhage-related morbidity differs in women exposed to methylergonovine or carboprost at cesarean delivery. They performed a secondary analysis using the Maternal-Fetal Medicine Units Network Cesarean Registry. They identified women who underwent cesarean delivery and received either methylergonovine or carboprost for refractory uterine atony. The primary outcome was hemorrhage-related morbidity defined as intraoperative or postoperative red blood cell transfusion or the need for additional surgical interventions including uterine artery ligation, hypogastric artery ligation, or peripartum hysterectomy for atony. They compared the risk of hemorrhage-related morbidity in those exposed to methylergonovine vs carboprost. Propensity-score matching was used to account for potential confounders. The study cohort comprised 1335 women; 870 (65.2\%) women received methylergonovine and $465(34.8 \%)$ women received carboprost. After accounting for potential confounders, the risk of hemorrhage-related morbidity was higher in the carboprost group than the methylergonovine group (relative risk, 1.7; 95\% confidence interval, 1.2-2.6). They conclude that methylergonovine was associated with reduced risk of hemorrhage-related morbidity during cesarean delivery compared to carboprost. Based on these results, methylergonovine may be a more effective second-line uterotonics [12].

To test the effect of misoprostol, Hofmeyer et al conclude that the misoprostol is not superior to the other uterotonics to prevent or treat PPH [13].

Morlando et al studied the change in the incidence of placenta accreta and associated risk factors along four decades. The incidence increased from $0.12 \%$ during the $1970 \mathrm{~s}$, to $0.31 \%$ during the $2000 \mathrm{~s}$. During the same period, cesarean section rates increased from 17 to $64 \%$. Prior cesarean section was the only risk factor showing a significant concomitant rise. The results reinforce cesarean section as the most significant predisposing condition for placenta accreta [14].

The prevention of hemorrhage in the third stage of delivery, in according to Umer et al (2014), is a challenge, in a review they made. The third stage of labor is associated with considerable maternal morbidity and mortality. The major complication is postpartum hemorrhage ( $\mathrm{PPH})$, which is the leading cause of maternal morbidity and mortality worldwide. Whereas in the event of PPH due to atony of the uterus there exist numerous treatment guidelines; for the management of retained placenta the general consensus is more difficult to establish. Active management of the third stage of labour is generally accepted as standard of care as already its duration is contributing to the risk of PPH. Despite scant evidence it is commonly advised that if the placenta has not been expelled
30 minutes after delivery, manual removal of the placenta should be carried out under anesthesia. Pathologic adhesion of the placenta in the low risk situation usually is diagnosed at the time of delivery; therefore a pre- or intrapartum screening opportunity for placenta accreta would be desirable. But diagnosis of abnormalities of placentation other than placenta previa remains a challenge. Nevertheless the use of ultrasound and Doppler sonography might be helpful in the third stage of labor. An improvement might be the implementation of standardized operating procedures for retained placenta which could contribute to a reduction of maternal morbidity [6].

A very rare condition, choriocarcinoma gestational after term pregnancy $(1: 160,000)$ was reported by Bratilla et al (2015) and reminds us to emphasisee the placental examination. This case must be remembered as follows: a 29year-old woman gravidity 2, parity 2, with metastatic choriocarcinoma after term pregnancy, diagnosed at four months after the delivery of a healthy baby. An episode of abundant vaginal bleeding occurred after four months from delivery. The local exam revealed a vaginal tumor whose pathological examination on biopsy samples was inconclusive. Subsequently, she was admitted in our clinic with abundant vaginal bleeding, severe anemia and fever. Abdominal ultrasonography revealed an intracavitary uterine tumor mass with signs of myometrial invasion to the uterine serosa, strong Doppler signal and moderate ascites. Pulmonary X-ray and computed tomography scan excluded extra pelvic tumor masses. The pretreatment human chorionic gonadotropin (HCG) level was 31,030 IU/mL and her FIGO risk factor score was 8 (high-risk group). Total hysterectomy with bilateral salpingo-oophorectomy and omentectomy was performed as an optimal cytoreduction. Postoperative remaining lesions were represented by the metastasis located in the lower two-thirds of the vagina. Histopathological examination revealed uterine choriocarcinoma with ovarian metastasis. Postoperative was initiated four courses of polychemotherapy. Case evolution was favorable, with the normalization of the beta HCG value in two months postoperative and complete remission of the vaginal metastasis in six weeks postoperative [15].

\section{Conclusion}

Those who work in an obstetrics emergency sector must be aware of bleeding, once it is the most frequent cause of maternal death in the developing countries. There is a critical timing to interfere with the evolution of the situation until it becomes irreversible. The surgical procedure must be performed to save lives and training in an immediate knowledge, skills, and confidence are necessary [16].

\section{References}

[1] Carroli G, Cuesta C, Abalos E, Gulmezoglu AM: Epidemiology of postpartum haemorrhage: a systematic review. Best Pract Res Clin Obstet Gynaecol 2008, 22 (6): 999-1012. 
[2] Rath WH: Postpartum hemorrhage--update on problems of definitions and diagnosis. Acta Obstet Gynecol Scand 2011, 90 (5): 421-428.

[3] Weeks A: The prevention and treatment of postpartum haemorrhage: what do we know, and where do we go to next? BJOG 2015, 122 (2): 202-210.

[4] Mehrabadi A, Hutcheon JA, Liu S, Bartholomew S, Kramer MS, Liston RM, Joseph KS, Maternal Health Study Group of Canadian Perinatal Surveillance S: Contribution of placenta accreta to the incidence of postpartum hemorrhage and severe postpartum hemorrhage. Obstet Gynecol 2015, 125 (4): 814821.

[5] Abdul-Kadir R, McLintock C, Ducloy AS, El-Refaey H, England A, Federici AB, Grotegut CA, Halimeh S, Herman $\mathrm{JH}$, Hofer S et al: Evaluation and management of postpartum hemorrhage: consensus from an international expert panel. Transfusion 2014, 54 (7): 1756-1768.

[6] Urner F, Zimmermann R, Krafft A: Manual removal of the placenta after vaginal delivery: an unsolved problem in obstetrics. J Pregnancy 2014, 2014: 274651.

[7] Binkowska M, Ciebiera M, Jakiel G: Placenta accreta: review and 3 case reports. Ginekol Pol 2015, 86 (5): 396-400.

[8] Takebayashi A, Kimura F, Yamanaka A, Takahashi A, Tsuji S, Ono T, Kaku S, Kita N, Takahashi K, Okabe H et al: Exaggerated placental site, consisting of implantation site intermediate trophoblasts, causes massive postpartum uterine hemorrhage: case report and literature review. Tohoku J Exp Med 2014, 234 (1): 77-82.

[9] Luo A, Mao P: Late postpartum hemorrhage due to placental and fetal membrane residuals: experience of two cases. Clin Exp Obstet Gynecol 2015, 42 (1): 104-105.

[10] Rivera-Rosado M, Flores-Perez IS, Mendez K, Rivera-Vinas JI: Risk factors for hysterectomy in abnormal placentation at the University District Hospital. Bol Asoc Med P R 2014, 106 (1): 27-29.

[11] Calvo-Aguilar O, Vasquez-Martinez J, Hernandez-Cuevas P: [Obstetric hysterectomy in the General Hospital Dr. Aurelio Valdivieso: three-year review]. Ginecol Obstet Mex 2016, 84 (2): $72-78$.

[12] Butwick AJ, Carvalho B, Blumenfeld YJ, El-Sayed YY, Nelson LM, Bateman BT: Second-line uterotonics and the risk of hemorrhage-related morbidity. Am J Obstet Gynecol 2015, 212 (5): 642 e 641-647.

[13] Hofmeyr GJ, Gulmezoglu AM, Novikova N, Lawrie TA: Postpartum misoprostol for preventing maternal mortality and morbidity. Cochrane Database Syst Rev 2013, 7: CD008982.

[14] Morlando M, Sarno L, Napolitano R, Capone A, Tessitore G, Maruotti GM, Martinelli P: Placenta accreta: incidence and risk factors in an area with a particularly high rate of cesarean section. Acta Obstet Gynecol Scand 2013, 92 (4): 457-460.

[15] Bratila E, Ionescu CA, Vladescu CT, Cirstoiu MM, Berceanu C: Gestational choriocarcinoma after term pregnancy: a case report. Rom J Morphol Embryol 2015, 56 (1): 267-271.

[16] Nelissen E, Ersdal H, Mduma E, Evjen-Olsen B, Broerse J, van Roosmalen J, Stekelenburg J: Helping Mothers Survive Bleeding After Birth: retention of knowledge, skills, and confidence nine months after obstetric simulation-based training. BMC Pregnancy Childbirth 2015, 15: 190. 